\title{
Ultrasound in the evaluation of penetrating thoraco-abdominal trauma: a review of the literature.
}

\author{
Nicholas J. Governatori ${ }^{1}$, Turandot Saul ${ }^{2}$, Sebastian D. Siadecki², Resa E. Lewiss ${ }^{3}$
}

${ }^{1}$ Thomas Jefferson University Hospital, Philadelphia PA, ${ }^{2}$ Department of Emergency Medicine, Division of Emergency Ultrasound, Mount Sinai St. Luke's Mount Sinai Roosevelt Hospital Center, New York, ${ }^{3}$ University of Colorado Hospital, Aurora CO USA

\begin{abstract}
The use of ultrasound in the evaluation of blunt thoraco-abdominal trauma is well described. Evidence for the use of ultrasound in the evaluation of penetrating cardio-thoracic and abdominal trauma, however, is more limited and varied. Current literature demonstrates that ultrasound is an excellent screening tool for penetrating thoracic and cardiac injuries with a high sensitivity for detecting injury requiring acute intervention. For abdominal injuries, however, the sensitivity for detection of injury is low and thus the utility of ultrasound as a screening tool is limited. This review summarizes the existing literature addressing the clinical utility of ultrasound for penetrating trauma to the pericardium, thorax and abdomen.
\end{abstract}

Keywords: ultrasound, echocardiography, hemopericardium, hemothorax, hemoperitoneum,

Ultrasound for the evaluation of blunt abdominal trauma was first described by Scandinavian interventional radiologist Kristensen et al in the early 1970s [1]. Twenty years later, in the 1990s, trauma surgeon Grace Rocyzki et al were using ultrasound as the primary modality for injured patient assessment [2]. Initially, called the FAST (focused abdominal sonography for trauma) examination, as the application expanded and it was incorporated into the Advanced Trauma Life Support Course, the acronym's meaning changed to the Focused Assessment for the Sonographic examination of the Trauma patient [3]. During this period, surgeons and emergency physicians rapidly adopted this noninvasive imaging method of evaluating trauma patients at the bedside $[2,4]$. Since then, the role of ultrasound in blunt trauma has been well studied. Level 1 evidence exists for the FAST examina-

Received 15.09.2015 Accepted 08.10.2015

Med Ultrason

2015, Vol. 17, No 4, 528-534

Corresponding author: Turandot Saul, MD

Mount Sinai St. Luke's Hospital

Mount Sinai Roosevelt Hospital

Department of Emergency Medicine

Division of Emergency Ultrasound

1000 Tenth Avenue, Room GE-01

New York, New York 10019

Phone: 212-523-3981, Fax: 212-523-2186

E-mail: turan@joshsaul.com tion to be considered the initial diagnostic modality to exclude hemoperitoneum in these patients [5-9]. Currently, practitioners accept the accuracy rate for ultrasound as nearly equivalent to that of diagnostic peritoneal lavage (DPL) and computed tomography (CT) [10-12]. However, the evidence is less clear for the use of ultrasound in the setting of penetrating thoraco-abdominal trauma.

This review presents the current literature on the role of ultrasound in the evaluation of a patient presenting with penetrating cardiac, pleural and peritoneal injury.

\section{Ultrasound for penetrating cardiac injury}

A 1980 study by DeGennero et al advocated immediate surgical intervention in all patients with "potential penetrating thoracic injuries in or near the cardiac silhouette". The authors based their recommendations upon a comparison of the clinical course of 10 trauma patients treated with observation and conservative management with 33 trauma patients who went immediately for operative intervention. Survival rates increased from $20 \%$ to $67 \%$ after the direct to the operating room policy was instituted [13]. Consequently, through the late 1980s, diagnostic sub-xiphoid pericardiotomy was performed to evaluate for a pericardial effusion following a penetrating injury to the thorax [14]. This approach was very reli- 
able but resulted in many negative procedures, one study reporting a rate of $76 \%$, and potential complications as high as $54 \%$ in another study $[15,16]$.

Ultrasound is very sensitive for the detection of fluid in the pericardial space. With optimal images and correct interpretation, as little as $20 \mathrm{cc}$ of fluid can be visualized [17]. A 1984 case report was one of the earliest publications to advocate for the use of ultrasound in penetrating trauma. A patient with a stab wound to the chest presented with a left hemothorax diagnosed on chest radiograph and was treated with tube thoracostomy. After discharge, the patient returned with repeated syncopal episodes and negative chest radiographs until a sonographic cardiac examination demonstrated hemopericardium [18]. The actual number of patients that develop late pericardial effusions is unknown [19].

A 1988 case report provided another example of the utility of cardiac ultrasound in penetrating trauma. A patient with a stab wound to the right upper chest was found to have right-sided pleural fluid on chest radiograph. After tube thoracostomy did not improve his clinical status, a cardiac ultrasound revealed a pericardial effusion and the patient was taken to the operating room [20]. The authors concluded that with a penetrating thoracic injury, ultrasound examination was able to provide a fast and efficient way to demonstrate pericardial fluid. Similar results were reported in a case of a ventral septal defect diagnosed after detection of hemopericardium on bedside ultrasound [21]. Ultrasound evaluation offered an alternative to observation versus the aggressive straight to the operating room approach.

Further research was needed to determine a clear role for ultrasound in penetrating thoracic trauma. In 1990 the first prospective study demonstrating the accuracy, sensitivity and specificity of ultrasound was published. In this study, 73 patients with penetrating thoracic trauma and stable vital signs received an ultrasound followed by a sub-xiphoid pericardial window in the operating room. Overall, ultrasound was found to have an accuracy of $96 \%$, specificity of $97 \%$, and sensitivity of $90 \%$ for predicting cardiac injury [14]. Importantly, the authors concluded that the selective use of sub-xiphoid pericardiotomy only in patients with a positive ultrasound could eliminate unnecessary surgical procedures.

A 1991 study from the University of California, Davis et al, yielded similar results. Thirty six hemodynamically stable patients with penetrating precordial trauma received a cardiology department ultrasound examination to determine their disposition: directly to the operating room (large effusion), Intensive Care Unit for 48 hours (small effusion) or ward bed (no effusion). There was no evidence of missed cardiac injury during hospitalization or after hospital discharge [22]. Ultimately, the authors concluded that the high negative predicative value made from ultrasound an excellent screening tool allowing prompt detection of cardiac injury in the stable patient.

In 1992, the first article was published involving a large group of emergency medicine physicians trained to perform this procedure [23]. This 10-year retrospective review of 49 patients found that point of care (POC) cardiac ultrasound not only decreased time to diagnosis and disposition to the operating room (15.5 minutes in the ultrasound group versus 42.4 minutes in the nonultrasound group), but also improved both the survival rate $(100 \%$ in the ultrasound group compared to $57 \%$ in the non-ultrasound group) and neurological outcomes. This was thought to be due to more rapid diagnosis and prompt surgical intervention. There were no false negative examinations on chart review of patient outcomes.

In 1995, a second retrospective chart review from Cook County Hospital examined 121 clinically stable patients with penetrating wounds in the proximity of the heart following an institutional policy change [24]. Patients with evidence of sonographic pericardial effusion by the cardiology fellow immediately underwent a sub-xiphoid pericardial window, while those without were admitted and observed for 24-48 hours. The overall sensitivity, specificity and accuracy of ultrasound was determined to be $96.8 \%, 100 \%$, and $99.2 \%$ respectively with a positive predictive value of $100 \%$ and a negative predictive value of $98.9 \%$. The number of sub-xiphoid windows was reduced from 105 to 15 during this period and no clinically significant injuries were missed.

Despite the overwhelmingly positive literature during this period, two articles were also published warning of concerning false negative cases. A 1993 case series emphasizes that a cardiac ultrasound without significant pericardial fluid did not necessarily rule out major injury [25]. The article presented five cases of hemodynamically stable patients with penetrating pericardial trauma, all who had major intra-pericardial injuries. The missed diagnoses were thought to be due to the operator dependent nature of ultrasound and the difficulty in obtaining cardiac views in patients with chest wounds and/or bandages.

Similarly, in a 1995 prospective study of 105 hemodynamically stable patients with penetrating thoracic trauma, Meyer et al reported that ultrasound performed by the attending surgeon had significant limitations in identifying serious cardiac injuries in patients with an associated hemothorax (initial chest radiographic evidence of fluid within the pleural cavity) [26]. When compared to sub-xiphoid exploration as the gold standard, POC ultrasound missed four significant cardiac injuries making it only $56 \%$ sensitive in these patients. Two poten- 
tial reasons were cited by the authors as explanations; first, an injury that lacerates the pericardium may allow decompression of intra-pericardial fluid into the pleural cavity, decreasing the accumulation of fluid collection at the pericardial-myocardial interface. Second, the complex echoes of adjacent pleural fluid/blood may alter the interpretability of the cardiac ultrasound.

Further research in the late 1990s seemed to reaffirm the utility of POC ultrasound. A 1996 study from Emory University achieved $100 \%$ sensitivity and specificity. In this study, surgeons performed pericardial ultrasound examinations in 247 hemodynamically stable patients with penetrating truncal wounds but no immediate indication for operative intervention [27]. Patients were followed during their admission, through discharge and as outpatients in clinic, and there here were no false negative or false positive studies. Unlike Meyer's findings, which suggested that ultrasound had limited sensitivity in patients with an associated hemothorax [26], four of the ten patients in this study with cardiac tamponade also had hemothoraces, and hemopericardium was easily identified on the initial pericardial ultrasound examination.

A larger multi-center study, which included prospectively collected data from cardiac ultrasound performed by surgeons or the cardiology department of five Level 1 trauma centers, was published in 1999 [28]. Patients with positive examinations underwent surgical exploration and those with negative examinations were admitted for observation and followed for a minimum of 23 hours. Of the pooled 261 patients, there were no false negative studies and seven false positive studies, all of which examined only a single subcostal view. Six of these patients had an associated hemothorax. Overall the sensitivity of ultrasound was $100 \%$, the specificity was $96.9 \%$ and the accuracy was $97.3 \%$ for detecting hemopericardium. Furthermore, the mean time from ultrasound to operating room was 12.1 minutes. The authors concluded the study stating they "routinely use ultrasound initially and almost exclusively for the evaluation of patients with penetrating injuries to the precordial or transthoracic region" and that the "indications for a sub-xiphoid pericardial window had narrowed substantially."

As outlined by a 2015 study, the high sensitivities achieved in prior studies may be related to the small numbers of patients with actual cardiac injuries. One hundred seventy two patients presenting with penetrating chest wounds were examined by ultrasound followed by a subxiphoid pericardial window. There were a total of eighteen false negatives: eleven had an associated hemothorax, six had pneumopericardium, while one of the patients returned with a delayed cardiac tamponade after two negative studies (overall sensitivity of $86.7 \%$ ).
The authors found that, if there is presence or suggestion of either hemothorax or pneumopericardium, then further diagnostic testing is required [29]. Similarly, a 2009 study, found that the numbers of false negatives were increased with concurrent lacerations of the pericardial sac secondary to sequestration of the blood into the thoracic cavity therefore preventing accumulation of a hemopericardium [30].

Both authors conclude that if none of these conditions are suspected, the patient may be safely discharged home. A 2013 report from Cook County warns against this policy, citing that it is challenging if not impossible to determine the true incidence of missed cardiac injuries through retrospective studies [31]. Without a doubt further studies are required to determine whether ultrasound is a safe and effective screen tool for penetrating cardiac injury.

\section{Ultrasound for penetrating pleural injury}

\section{Hemothorax}

Ultrasound can serve as a diagnostic tool for the detection of hemothorax in penetrating thoracic injury. It is estimated that 50-100 $\mathrm{cc}$ of pleural fluid can be detected on upright chest radiograph [32], while $175 \mathrm{cc}$ is usually necessary for detection when the radiograph is taken with the patient supine [33]. Even smaller quantities of fluid can be detected with ultrasound, with one study estimating that as little as $20 \mathrm{cc}$ of pleural fluid can be visualized [34].

In 1995, Ma et al compared emergency medicine physician-performed thoracic ultrasound for free pleural fluid in 240 trauma patients to a gold standard of chest radiograph or tube thoracostomy drainage. In the 25 patients with hemothorax (from both blunt and penetrating injuries) all were detected by ultrasound and there were no false negative examinations in the patients with penetrating trauma [10].

Several of the same authors conducted a further study in 1997 comparing emergency medicine physician-performed ultrasound to the gold standard of computed tomography, tube thoracostomy or both [35]. Ultrasound accurately detected all 18 cases of hemothorax from penetrating thoracic injury. There were no false positive findings resulting in a sensitivity and specificity of $100 \%$.

\section{Pneumothorax}

Multiple studies have evaluated the test characteristics of ultrasound for the detection of pneumothorax in blunt thoracic trauma with sensitivities ranging from $92-100 \%$ and specificities ranging from $94-99 \%$ when compared to a gold standard of computed tomography [36-38]. Several studies have also evaluated the test char- 
acteristics of ultrasound for pneumothorax in the setting of penetrating trauma. In 2001, Dulchavsky et al compared surgeon-performed thoracic ultrasound to chest radiograph findings as a gold standard. In the 83 cases of penetrating thoracic trauma, 15 patients had a radiographic pneumothorax, all of which were detected by ultrasound, for a sensitivity and specificity of $100 \%$. It was noted that in two cases the pleural interface could not be visualized due to the presence of subcutaneous air [39]. A 2004 study by Knudtson et al also compared surgeonperformed ultrasound to findings on chest radiograph in 21 patients and reported the sensitivity, specificity, positive predictive value, negative predictive value, and accuracy for pneumothorax to all be $100 \%$ [40]. The authors concluded from their findings that ultrasound could be used as an adjunct or precursor to chest radiography in the evaluation of the trauma patient.

A 2012 study by $\mathrm{Ku}$ et al compared thoracic ultrasound for pneumothorax by both surgeons and emergency medicine physicians to a more robust gold standard of computed tomography, tube thoracostomy output or supine chest radiograph followed by clinical observation. In 47 patients with pneumothorax (blunt and penetrating), ultrasound had a sensitivity of $57 \%$ and a specificity of $99 \%$. The authors contributed the poor sensitivity to the diverse and varied prior ultrasound experience of their sonographer group, recognizing that most other previous studies were performed by a small number of highly trained sonographers [41].

Evaluating thoracic ultrasound for pneumothorax due to all causes, a 2012 meta-analysis by Alrajhi et al pooled data from 8 studies ( 6 trauma studies [both blunt and penetrating] and 2 iatrogenic) that all used computed tomography or tube thoracostomy drainage as the gold standard. Overall, the authors reported a sensitivity of $90.9 \%$, a specificity of $98.2 \%$, a positive predictive value of $94.4 \%$, and a negative predictive value of $97.0 \%$ [42]. Ultrasound had excellent test characteristics for the detection of pneumothoraces in this large population of mixed etiology.

\section{Ultrasound for penetrating thoraco-abdominal injury}

Initially, most studies in penetrating trauma focused on pericardial fluid, but later, several studies evaluated its utility in abdominal trauma as well. A study by Ma et al in 1995 prospectively evaluated the use of a rapid trauma ultrasound examination in blunt and penetrating torso trauma patients. After emergency medicine residents, fellows and faculty underwent 10 hours of didactic instruction, case review and hands-on sessions, they were able to detect pericardial fluid with a sensitivity of $100 \%$ and a specificity of $99 \%$ and detected intra-peritoneal fluid with a sensitivity of $86 \%$ and a specificity of $99 \%$ when compared to the gold standards of CT, diagnostic peritoneal lavage, radiograph, operative findings, or cardiology department echocardiography. They concluded that regardless of the performing physician's specialty, the more important details were immediate availability and a desire to learn how to perform a trauma ultrasound examination [10].

A 2001 study from the University of Maryland examined 75 consecutive stable patients with penetrating injuries to the abdomen, back and flank. Twenty two of 54 patients with a surgeon-performed negative FAST had intra-abdominal injury at laparotomy yielding an overall sensitivity of $46 \%$. There were two false positives (a hepatic vein thought to be free fluid and pleural fluid thought to be peritoneal fluid) yielding a specificity of 94\%. When compared to other modalities, the FAST examination had the highest specificity and positive predictive value whereas computerized tomography (CT) had the highest accuracy [43]. The authors concluded that a positive FAST warranted immediate surgical intervention, whereas a negative study required further diagnostic imaging.

A similarly designed prospective observational study, also in 2001, demonstrated a slightly increased sensitivity of $67 \%$ with a specificity of $98 \%$ for the use of technician-performed sonography in penetrating torso injury. Despite the high specificity for detecting pericardial or peritoneal fluid, 6 of the 53 initially negative examinations had a significant abdominal injury detected by further diagnostic testing, 5 of which required surgical repair. The authors concluded that ultrasound should be used as one component of a diagnostic algorithm and should not be the definitive test in all cases [44].

A 2004 prospective observational study from Carolinas Medical Center reported a 100\% sensitivity and specificity for evaluation of traumatic pericardial effusion and intra-peritoneal fluid. Thirty-two hemodynamically stable patients with penetrating trauma were evaluated by emergency medicine physicians and trauma surgeons. Of the 16 patients whose ultrasound was initially negative, no other diagnostic imaging revealed any further injury nor did any return to the hospital within six months with a missed injury. Although the high sensitivity in detecting hemopericardium had been reproduced in prior studies, this study was the first to show $100 \%$ sensitivity and $100 \%$ specificity for detecting intra-peritoneal injury [45].

Ultrasound does not usually detect the actual solid or visceral organ injury but rather relies on the evaluation of 
free fluid in potential spaces as a marker of injury. A 2004 study noted that the presence of free peritoneal fluid does not always necessitate therapeutic intervention [46,47]. Thirty-eight patients with penetrating abdominal trauma underwent FAST examination by a surgeon and the results were compared to CT, operative findings, autopsy report, and hospital course. Ultrasound had a sensitivity of $91.7 \%$ and specificity of $100 \%$ for detecting free fluid, however, the sensitivity decreased to $71.4 \%$ with a specificity of $95.8 \%$ in the detection of cases that required therapeutic intervention.

As an alternative to evaluating for free fluid, one study used a direct comparison of edema surrounding the muscle bundle and fascia at the site of the injury by surgeons and emergency medicine physicians. They demonstrated a sensitivity and negative predictive value of $100 \%$ when compared to chest radiograph/diagnostic peritoneal lavage/CT scan depending on location [48]. The specificity and positive predictive values, however, were $48.7 \%$, in contrast to the traditional FAST examination, which typically carries a high specificity [43]. The authors concluded that a simple side-by-side comparison of injured abdominal wall layers may help to increase the sensitivity of POC ultrasound.

\section{Discussions}

Ultrasound is the initial diagnostic tool for blunt abdominal trauma. It has additionally become an important tool in the evaluation of penetrating cardiac and thoracic injury. The 2008 American College of Emergency Physicians Emergency Ultrasound Guidelines states that "[ultrasound] applies to both blunt and penetrating trauma in all ages" [49].

Difficulties in the performance and interpretation of cardiac ultrasound in penetrating trauma appear to be in patients with a co-existent hemothorax $[26,28]$ air in the pericardial sac [29], and also lacerations of the pericardial sac [30]. This may be because the fluid accumulates into the thoracic cavity rather than the pericardium, or that it the hemothorax obscures the pericardium and makes differentiation of anatomical planes difficult. Some authors suggest a role for repeat ultrasonography after an initial negative examination [19]. Without a doubt, care should be taken when performing cardiac ultrasound in this patient population and further prospective trials are necessary to determine the true accuracy to detect cardiac injuries in patients with concurrent injuries [31].

The utility of ultrasound in evaluating penetrating abdominal injury is variable. Although some studies have shown to be favorable $[40,46,50]$ the data is still lacking. Ultrasound relies on the evaluation of free fluid in potential spaces as a marker of injury, not necessarily identification of the injury itself, leading to sensitivities ranging from $46-100 \%$, specificities from $48-100 \%$, and negative predictive values from $60-98 \%$. The data is further complicated by the lack of standardization of anatomical landmarks between ultrasound scan protocols across studies.

\section{Conclusion}

This article summarizes the existing literature addressing the clinical utility of ultrasound for penetrating trauma. Current literature demonstrates that ultrasound is an excellent screening tool for penetrating thoracic and cardiac injuries with a high sensitivity for detecting injury requiring acute intervention. For abdominal injuries, however, the sensitivity for detection of injury is low and thus the utility of ultrasound as a screening tool is limited.

\section{Conflict of interest: none}

\section{References}

1. Kristensen JK, Buemann B, Kühl E. Ultrasonic scanning in the diagnosis of splenic haematomas. Acta Chir Scand 1971; 137: 653-657.

2. Rozycki GS, Ochsner MG, Schmidt JA, et al. A prospective study of surgeon-performed ultrasound as the primary adjuvant modality for injured patient assessment. J Trauma 1995; 39: 492-498.

3. Rozycki GS, Shackford SR. Ultrasound, what every trauma surgeon should know. J Trauma 1996; 40: 1-4.

4. Mateer J, Plummer D, Heller M, et al. Model curriculum for physician training in emergency ultrasonography. Ann Emerg Med 1994; 23: 95-102.

5. Boulanger BR, Brenneman FD, Kirkpatric AW, McLellan BA, Nathens AB. The indeterminate abdominal sonogram in multisystem blunt trauma. J Trauma 1998; 45: 52-56.

6. Bode PJ, Edwards MJ, Kruit MC, van Vugt AB. Sonography in a clinical algorithm for early evaluation of 1671 patients with blunt abdominal trauma. AJR Am J Roentgenol 1999; 172: 905-911.

7. McKenney MG, Martin L, Lentz K, et al. 1,000 consecutive ultrasound for blunt abdominal trauma. J Trauma 1996; 40: 607-612.

8. Hoff WS, Holevar M, Nagy KK, Patterson L, Young JS, Arrillaga A et al. Practice management guidelines for the evaluation of blunt abdominal trauma: the EAST practice management guidelines work group. J Trauma 2002; 53: 602-615.

9. Symbas NP, Bongiorno PF, Symbas PN. Blunt cardiac rupture: the utility of emergency department ultrasound. Ann Thorac Surg 1999; 67: 1274-1276.

10. Ma OJ, Mateer JR, Ogata M, Kefer M, Whittmann D, Aprahamian C. Prospective analysis of a rapid trauma ul- 
trasound examination performed by emergency physicians. J Trauma 1995; 38: 879-885.

11. Boulanger BR, McLellan BA, Brenneman FD, et al. Emergent abdominal sonography (EAS) as a screening test in a new diagnostic algorithm for blunt trauma. J Trauma 1996; 40: 867-874.

12. Patel AN, Brennig C, Cotner J, et al. Successful diagnosis of penetrating cardiac injury using surgeon-performed sonography. Ann Thorac Surg 2003; 76: 2043-2047.

13. DeGennaro VA, Bonfils-Roberts EA, Ching N, Nealon TF Jr. Aggressive management of potential penetrating cardiac injuries. J Thorac Cardiovasc Surg 1980; 79: 833-837.

14. Jimenez E, Martin M, Krukenkamp I, Barrett J. Subxiphoid pericardiotomy versus echocardiography: a prospective evaluation of the diagnosis of occult penetrating cardiac injury. Surgery 1990; 108: 676-679.

15. Duncan AO, Scalea TM, Sclafani SJ, et al. Evaluation of occult cardiac injuries using subxiphoid pericardial window. The Journal of Trauma 1989; 29: 955-960.

16. Demetriades D, Van Der Veen BW. Penetrating injuries of the heart: experience over two years in South Africa. J Trauma 1989; 23: 1034-1041.

17. Horowitz MS, Schultz CS, Stinson EB, Harrison DC, Popp RL. Sensitivity and specificity of echocardiographic diagnosis of pericardial effusion. Circulation 1974; 50: 239-247.

18. Choo MH, Chia BL, Chia FK, Johan A. Penetrating cardiac injury evaluated by two-dimensional echocardiography. Am Heart J 1984; 108: 417-420.

19. Simmons JD, Haraway AN, Schmieg RE Jr, Burgdorf M, Duchesne J. Is there a role for secondary thoracic ultrasound in patients with penetrating injuries to the anterior mediastinum? Am Surg 2008; 74: 11-14.

20. Whye D, Barish R, Almquist T, Groleau G, Tso E, Browne B. Echocardiographic diagnosis of acute pericardial effusion in penetrating chest trauma. Am J Emerg Med 1988; 6: 21-23.

21. Jeon K, Lim WH, Kang SH, et al. Delayed diagnosis of traumatic ventricular septal defect in penetrating chest injury: small evidence on echocardiography makes big difference. J Cardiovasc Ultrasound 2010; 18: 28-30.

22. Freshman SP, Wisner DH, Weber CJ. 2-D echocardiography: emergent use in the evaluation of penetrating precordial trauma. J Trauma 1991; 31: 902-905.

23. Plummer D, Brunette D, Asinger R, Ruiz E. Emergency department echocardiography improves outcome in penetrating cardiac injury. Ann Emerg Med 1992; 21: 709-712.

24. Nagy KK, Lohmann C, Kim DO, Barrett J. Role of echocardiography in the diagnosis of occult penetrating cardiac injury. J Trauma 1995; 38: 859-862.

25. Bolton JW, Bynoe RP, Lazar HL, Almond CH. Two-dimensional echocardiography in the evaluation of penetrating intrapericardial injuries. Ann Thorac Surg 1993; 56): 506-509.

26. Meyer DM, Jessen ME, Grayburn PA. Use of echocardiography to detect occult cardiac injury after penetrating thoracic trauma: a prospective study. J Trauma 1995; 39: 902-907.
27. Rozycki GS, Feliciano DV, Schmidt JA, et al. The role of surgeon-performed ultrasound in patients with possible cardiac wounds. Ann Surg 1996; 223: 737-744.

28. Rozycki GS, Feliciano DV, Ochsner MG, et al. The role of ultrasound in patients with possible penetrating cardiac wounds: a prospective multicenter study. J Trauma 1999; 46: 543-551.

29. Nicol AJ, Navsaria PH, Benengfield S, Hommes M, Kahn D. Screening for occult penetrating cardiac injuries. Ann Surg 2015; 261: 573-578.

30. Ball, CG, Williams BH, Wyrzykowski AD, Nicholas JM, Rozycki GS, Feliciano DV. A caveat to the performance of pericardial ultrasound in patients with penetrating cardiac wounds. J Trauma 2009; 67: 1123-1124.

31. Cull JD, Bokhari F. Penetrating chest injury in the setting of a hemothorax: should pericardial windows replace echocardiograms? Am Surg 2013;79: E56-E57.

32. Rubens MB. The pleura: Collapse and consolidation, in Sutton D (ed.). A Textbook of Radiology Imaging. $4^{\text {th }}$ ed. Edinburgh: Churchill Livingstone, 1987: 393.

33. Juhl JH. Diseases of the pleura, mediastinum and diaphragm. In: Juhl JH, Crummy AB (eds). Essentials of Radiologic Imaging. $6^{\text {th }}$ ed. Philadelphia, Lippincott, 1993: 1026.

34. Rothlin MA, Naf R, Amgwerd M, Candinas D, Frick T, Trentz O. Ultrasound in blunt abdominal and thoracic trauma. J Trauma 1993; 34: 488-495.

35. Ma OJ, Mateer JR. Trauma ultrasound examination versus chest radiography in the detection of hemothorax. Ann Emerg Med 1997; 29: 312-316.

36. Rowan KR, Kirkpatrick AW, Liu D, Forkheim KE, Mayo JR, Nicolaou S. Traumatic pneumothorax detection with Thoracic US: Correlation with chest radiograph and CT initial experience. Radiology 2002; 225: 210-214.

37. Blaivas M, Lyon M, Duggal S. A prospective comparison of supine chest radiography and bedside ultrasound for the diagnosis of traumatic pneumothorax. Acad Emerg Med $2005 ; 12: 844-849$.

38. Soldati G, Testa A, Sher S, Pignataro G, La Sala M, Silveri NG. Occult traumatic pneumothorax. Chest 2008; 133 : 204-211.

39. Dulchavsky SA, Schwarz KL, Kirkpatrick AW, et al. Prospective evaluation of thoracic ultrasound in the detection of pneumothorax. The J Trauma 2001; 50: 201-205.

40. Knudtson JL, Dort JM, Helmer SD, Smith RS. Surgeon performed ultrasound for pneumothorax in the trauma suite. J Trauma 2004; 56: 527-530.

41. Ku BS, Fields JM, Carr B, Everette WW, Gracias VH and Dean AJ. Clinician performed bedside ultrasound for the diagnosis of traumatic pneumothorax. West J Emerg Med 2012; 14: 103-108.

42. Alrajhi K, Woo MY, Vaillancourt C. Test characteristics of ultrasonography for the detection of pneumothorax: a systematic review and meta-analysis. Chest 2012; 141: 703-708.

43. Udobi KF, Rodriguez A, Chiu WC, Scalea TM. Role of ultrasonography in penetrating abdominal trauma: a prospective clinical study. J Trauma 2001; 50: 475-479. 
44. Boulanger BR, Kearney PA, Tsuei B, Ochoa JB. The routine use of sonography in penetrating torso injury is beneficial. J Trauma 2001; 51: 320-325.

45. Tayal VS, Beatty MA, Marx JA, Tomaszewski CA, Thomason MH. FAST (focused assessment with sonography in trauma) accurate for cardiac and intraperitoneal injury in penetrating anterior chest trauma. J Ultrasound Med 2004; 23: 467-472.

46. Kirkpatrick AW, Sirois M, Ball CG, et al. The hand-held ultrasound examination for penetrating abdominal trauma. Am J Surg 2004; 187: 660-665.

47. O'Dochartaigh D, Douma M. Prehospital ultrasound of the abdomen and thorax changes trauma patient manage- ment: A systematic review. Injury 2015. doi: 10.1016/j.injury.2015.07.007.

48. Bokhari F, Nagy K, Roberts R, et al. The ultrasound screen for penetrating truncal trauma. Am Surg 2004; 70: 316-321.

49. American College of Emergency Physicians. Emergency Ultrasound Guidelines 2008. Policy statement. Available at: http://www.acep.org

50. Gillman LM, Ball CG, Panebianco N, Al-Kadi A, Kirkpatrick AW. Clinician performed resuscitative ultrasonography for the initial evaluation and resuscitation of trauma. Scand J Trauma Resusc Emerg Med 2009; 17: 34. 